\title{
Co-optimization approach to post-storm recovery for interdependent power and transportation systems
}

\author{
Yinyin GE ${ }^{1}$, Lili $\mathrm{DU}^{2}$, Hongxing $\mathrm{YE}^{3}$
}

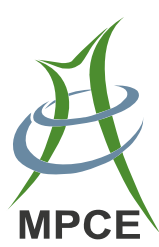

\begin{abstract}
The power and transportation systems are urban interdependent critical infrastructures (CIs). During the post-disaster restoration process, transportation mobility and power restoration process are interdependent, and their functionalities significantly affect other well-beings of other urban CIs. Therefore, to enhance the resilience of urban CIs, successful recovery strategies should promote CI function cooperatively and synergistically to distribute goods and services efficiently. This paper develops an integrative framework that addresses the challenges of enhancing the recovery efficiency of urban power and transportation systems in short-term recovery period. Specifically, the post-storm recovery process is considered as a scheduling problem under the constraints representing crew dispatch, equipment and fuel limit. We propose a new framework for co-optimizing the recovery scheduling of power and transportation systems, respecting precedency requirement and network constraints. The advantages and benefits of co-optimized recovery scheduling are validated in a testing system.
\end{abstract}

CrossCheck date: 25 January 2019

Received: 19 November 2018 / Accepted: 25 January 2019/Published online: 24 April 2019

(C) The Author(s) 2019

$\triangle$ Hongxing YE

h.ye@csuohio.edu

Yinyin GE

yxg417@case.edu

Lili DU

lili.du@essie.ufl.edu

1 Case Western Reserve University, Cleveland, USA

2 University of Florida, Gainesville, USA

3 Cleveland State University, Cleveland, USA
Keywords Co-optimization, Interdependent critical infrastructure (CI), Power system, Resilience,

Transportation system

\section{Introduction}

Among the most devastating natural hazards is coastal flooding caused by extreme storm events that interrupt critical infrastructures (CIs) in coastal cities, including building damage, roadway washout, power outage, gas shortage, communication disruption, etc., all of which lead to significant economic losses. For example, Hurricane Irma left around 6.2 million customers without power in Florida [1]. Flooding, debris caused numerous road closure around Northeast Florida and portions of I-4 washed out. The devastating effects of Hurricanes Harvey and Irma are estimated to cause economic loss between $\$ 42.5$ billion to $\$ 65$ billion [2].

With continuous rapid urbanization and growing population in coastal zones, anthropogenic changes make the coastal cities and coastal infrastructures more vulnerable to damage from extreme storms. Both the intensity and frequency of extreme storm events are expected to increase because of the climate change [3], and coastal flooding is expected to worsen in the future. Therefore, it is critically essential to enhance the resilience of CIs against extreme storm. In the short term, it is necessary to support emergency operations and the delivery of essential supplies. Debris on the main roadway need be cleared, then equipment and crews could be transported to restore power systems. Power restoration efforts should be steadily progressing to ensure other electricity-enable CIs are operational in the short term and intermediate term. Without electricity, providing transportation services can be a 
challenge as electricity powers the traffic signaling, switches, and gas stations. Power supply and traffic efficiency will significantly affect functions and restoration of other CI systems, such as water and communication systems. Therefore, to enhance the resilience of urban CIs, successful recovery strategies should promote interdependent critical infrastructures (ICIs) (such as roadway and power) function cooperatively and synergistically to distribute goods and services efficiently.

There is rich power literature on resilience against disaster, such as component hardening, cascading failures, resilience enhanced by microgrid, etc. For example, [4] presents some examples from different parts of the world where distributed energy resources in a microgrid were used to provide reliable electricity supply in the wake of disasters, allowing recovery and rebuilding efforts to occur with relatively greater efficiency. Reference [5] introduces strategies for microgrid operation when it becomes islanded. Other strategies for distribution system restoration are proposed in [6-12], while planning on power restoration in transmission systems are studied in [9, 13, 14]. Reference [15] reveals the need to strengthen electric infrastructure to minimize storm damage, reduce outages, and lessen restoration time with the need to mitigate excessive cost increases to electric customers. On-site generation in microgrid showed benefits of reliability during Hurrican Sandy [10]. The estimated annual cost due to weatherrelated outage ranges from $\$ 18$ billion to $\$ 70$ billion between 2003 and 2012 according to [12], which also describes strategies for modernizing the grid and increasing grid resilience. Reference [16] presents a detailed review for methods and tools of forecasting natural disaster related power system disturbances, hardening and pre-storm operations, and restoration models.

Transportation system provides the network to support the mobility of goods as well as personnel. In transportation engineering, many efforts have also been devoted to the research on transportation infrastructure systems in disasters [17]. Different techniques, such as analytical models, simulation and optimization models are applied for pre- and post-event assessment or management purposes [18-21]. Analytical methods are often used to analyze potential failure risks based on probabilities. Monte Carlo simulation-based methods involve a large sample of scenarios [22]. Optimization models optimize road network performance function, such as flow via pre-disaster network design or post-disaster resource allocation $[23,24]$.

In transportation sector, literature often emphasizes on the traffic mobility only. In the energy sector, coordinated operation of power and natural gas systems has attracted many attention due to their interdependency with the rise of natural gas-fired generators [25-27]. However, in the context of interdependency of energy and transportation systems, which belong to two different sectors, existing literature mostly aims at the electric vehicles and charging stations that are naturally connected to distribution network [28-30]. The interdependency between energy and transportation is indeed beyond electrical vehicles, especially in the aftermath of disasters.

The main contribution of this paper is to develop an integrative framework that addresses the challenges of enhancing the recovery efficiency of urban power and transportation systems in short-term recovery period. Although the recovery activities are synergistic and interdependent in power and transportation system, challenge of interdependency is seldom addressed in the post-storm recovery literature. In this paper, the post-storm recovery process is considered as a scheduling problem with constraints representing crew dispatch, equipment and fuel limit, and other resource sharing as well as constraints representing precedency relationship among the repair tasks. We propose a new framework for co-optimizing the repair scheduling in power and transportation systems.

The paper is organized as follows. Section 2 introduces the power and transportation systems and the recovery model. Section 3 demonstrates a case study for the proposed methods. Section 4 concludes the paper.

\section{Recovery model}

Post-storm recovery tasks are not independent to another. Some tasks have hard precedency relationship while others might share repair resources. For example, in order to repair components in power systems, such as generators, overhead and underground cables (lines), we have to guarantee the delivery of crew, fuel, and other resources. In this section, we will develop a co-optimization model for recovery activity scheduling in power and transportation systems.

An example of interdependent power and transportation systems is shown in Fig. 1. It consists of electricity distribution system and transportation system. We consider the electricity distribution system and the city roadway system. The electricity distribution system includes nodes, distributed generators, and transformers, while the transportation system consists of traffic intersections and roadways. It presents close topology and flow interdependency among power and transportation systems as the power nodes are often co-located around traffic intersections. Specifically, the repair of the components of power grid, after the failure caused by storms, will reply on the repair resources transported via roadways; on the other hand, the repair work of electrical components indeed will have significant impacts on the traffic flow, even cause some 


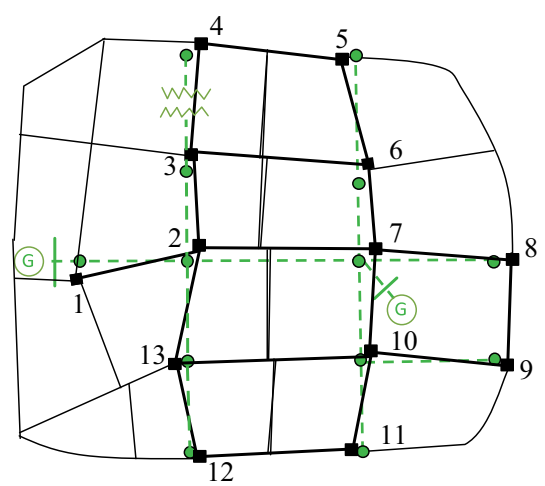

- - - Electricity distribution network; ○ Node; (G) Distributed generator

— District boundary; — Roadway network; - Traffic intersection Transformer

Fig. 1 An example of interdependent power and transportation systems

roadway closure. In this paper, we mainly focus on the energy recovery and road repair.

\subsection{Objective of recovery}

To implement the strategy discussed above, we develop a mixed-integer linear programming (MILP) model to formulate power flows, the interdependency of power and transportation systems, and crew/resource delivery. The objective is formulated as:

$\max \left[\alpha \sum_{i} \sum_{t} D_{i, t}+(1-\alpha) \sum_{r} \sum_{t} y_{r, t}\right]$

where $D_{i, t}$ is the load in node $i$ at time $t ; y_{r, t}$ is the indicator of road $r$ being cleared at time $t$; and $\alpha$ is the weight coefficient.

The objective is to restore load and clear road as much as possible. As both power and transportation systems are involved, we employ weight factor $\alpha$ to simplify the problem. In the extreme case, one could maximize recovered power only by setting set $\alpha=1$.

\subsection{Recovery of roadway}

The crew and resources are transported to repair the blocked roadways. In this model, we consider certain amount of resources are required for clearing roads. The recovery of road is formulated as:

$y_{r, t} \geq y_{r, t-1} \quad \forall r, t$

$\sum_{\tau=1}^{t}\left(R_{m(r), \tau}+R_{n(r), \tau}\right) \geq \hat{R}_{r} y_{r, t} \quad \forall r, t$

$y_{r, t} \in\{0,1\} \quad \forall r, t$ where $m(r)$ and $n(r)$ are the intersections connect to road $r$; $R_{m(r), t}$ and $R_{n(r), t}$ are the labor resources available for road $r$; and $\hat{R}_{r}$ is the total labor resources needed to repair the road $r$.

For simplicity, the resource is assumed available for the repair once it arrives at intersection that connects to the damaged roads or is nearby out-of-service power equipment. One could always add artificial intersection near the damaged roads if accuracy is needed. Equation (2) indicates that a road is always clear once it is repaired. Equation (3) represents that the road $r$ will be only cleared after the accumulated resources reach the required amount $\hat{R}_{r}$. Equation (4) means $y_{r, t}$ is a binary variable. The road $r$ is clear or recovered post-storm when $y_{r, t}$ is 1 , and is closed when $y_{r, t}$ is 0 .

\subsection{Recovery of electricity distribution line}

When there are line outages in the electricity distribution system, the recovery of electricity distribution line could also be modeled via binary variables. We model the line recovery as:

$$
\begin{aligned}
& u_{l, t} \geq u_{l, t-1} \quad \forall l, t \\
& \sum_{\tau=1}^{t} L_{l, \tau} \geq \hat{L}_{l} u_{l, t} \quad \forall l, t \\
& u_{l, t} \in\{0,1\} \quad \forall l, t
\end{aligned}
$$

where $u_{l, t}$ is the indicator of line $l$ status; $L_{l, \tau}$ is the labor resources that used for line repair; and $\hat{L}_{l}$ is the labor resources needed to repair line $l$.

According to (5), the line is always in normal condition once it is repaired. Equation (6) enforces the status of line $l$ at period $t$. Only the labor resources that used for line repair reach the amount $\hat{L}_{l}$, line $l$ can be back to normal operation. The line $l$ is in normal condition or recovered at period $t$ when $u_{l, t}$ is 1 , and is not in service when it is 0 .

\subsection{Interdependent power and transportation systems}

A key point of post-storm recovery is to consider the interdependency of the power and transportation systems. We have modeled the recovery actions for roads and cables in previous two subsections. Although both power system and transportation system are networked, the delivery mechanisms are different. More specifically, the delivery of electric power is near light-speed in electricity distribution system, while the delivery of crew and fuel via transportation sub-system has delay. Energizing components in distribution system relies on the availability of the 
equipment and fuel that transported via the road network. For simplicity, the resource is assumed available for repair once it is nearby out-of-service power equipment. The model of interdependent power and transportation systems for recovery is formulated as follows.

$$
\begin{aligned}
& R_{m, t}=R_{m, t-1}-\sum_{r \in \mathcal{F}(m)} F L_{r, t}^{R+}-\sum_{r \in \mathcal{T}(m)} F L_{r, t}^{R-} \\
& +\sum_{r \in \mathcal{T}(m)} F L_{r, t-\gamma(r)}^{R+} \\
& +\sum_{r \in \mathcal{F}(m)} F L_{r, t-\gamma(r)}^{R-} \quad \forall m, t \\
& 0 \leq F L_{r, t}^{R-} \leq M y_{r, t} \quad \forall r, t \\
& 0 \leq F L_{r, t}^{R+} \leq M y_{r, t} \quad \forall r, t \\
& L_{m, t}=L_{m, t-1}-\sum_{r \in \mathcal{F}(m)} F L_{r, t}^{L+}-\sum_{r \in \mathcal{T}(m)} F L_{r, t}^{L-} \\
& +\sum_{r \in \mathcal{T}(m)} F L_{r, t-\gamma(r)}^{L+} \\
& +\sum_{r \in \mathcal{F}(m)} F L_{r, t-\gamma(r)}^{L-} \quad \forall m, t \\
& 0 \leq F L_{r, t}^{L-} \leq M y_{r, t} \quad \forall r, t \\
& 0 \leq F L_{r, t}^{L+} \leq M y_{r, t} \quad \forall r, t \\
& F_{m, t}=F_{m, t-1}-\sum_{i \in \mathcal{G}(m)} \omega_{i} P_{i, t}-\sum_{r \in \mathcal{F}(m)} F L_{r, t}^{F+} \\
& -\sum_{r \in \mathcal{T}(m)} F L_{r, t}^{F-}+\sum_{r \in \mathcal{T}(m)} F L_{r, t-\gamma(r)}^{F+} \\
& +\sum_{r \in \mathcal{F}(m)} F L_{r, t-\gamma(r)}^{f-} \quad \forall r, t \\
& 0 \leq F L_{r, t}^{F-} \leq M y_{r, t} \quad \forall r, t \\
& 0 \leq F L_{r, t}^{F+} \leq M y_{r, t} \quad \forall r, t
\end{aligned}
$$

where $F L_{r, t}^{R+}$ and $F L_{r, t}^{R-}$ are the flows of road-repair resource in positive and negative directions, respectively; $F L_{r, t}^{L+}$ and $F L_{r, t}^{L-}$ are the flows of line-repair resource in positive and negative directions, respectively; $F L_{r, t}^{F+}$ and $F L_{r, t}^{F-}$ are the flows of distributed generator fuel in positive and negative directions, respectively; $\mathcal{F}(m)$ is the set of roads whose defined source intersection is $m ; \mathcal{T}(m)$ is the set of roads whose defined destination intersection is $m ; \mathcal{G}(m)$ is the set of generators that are accessed via intersection $m ; \gamma(r)$ is the time of delivering crew/resource in road $r ; R_{m, t}$ is the road-repair resource available at intersection $m ; L_{m, t}$ is the line-repair resource available at intersection $m ; F_{m, t}$ is the fuel arrived at intersection $m ; P_{i, t}$ is the generation output of unit $i ; \omega$ is the fuel-power coefficient; and $M$ is a big number.
The repair resource and fuel flows, i.e., $F L_{r, t}^{R+}, F L_{r, t}^{R-}$, $F L_{r, t}^{L+}, F L_{r, t}^{L-}, F L_{r, t}^{F+}$, and $F L_{r, t}^{F-}$ in (11)-(16), all go through the road network. Hence, transporting time for repair resource and fuel must be considered, and it is modeled in flow constraints (8), (11), and (14). We model flows in two directions separately so that the arriving time, leaving time, and transporting time could be handled independently in the networked system. Equation (8) stands for the roadrepair resource available in $m$ at period $t$ considering the resource leaving and arriving $m$ at $t$. Due to the transporting time, goods flow $F L_{r, t-\gamma(r)}^{R+}$ arriving $r$ at $t$ indeed left its source intersection at $t-\gamma(r)$. Hence, line-repair resource at period $t$ is a function of line-repair resource in last period, i.e. $t-1$, leaving resource and arriving resource at $t$. Similarly, the line-repair resource and fuel transportations are modeled in (11) and (14), respectively.

All goods flows are limited by road network capacity in (9), (10), (12), (13), (15), and (16). For example, if $y_{r, t}$ is 0 , then (9) enforces the flow $F L_{r, t}^{R-}$ be zero at time $t$. In other words, if road $r$ is not cleared, the goods cannot be transported via $r$. The fuel consumption is modeled in (14), i.e. generating at level of $P_{i, t}$ will consume fuel at level of $\omega P_{i, t}$. At intersection $m$ in road network, the available fuel at $t$ is a function of fuel level at $t-1$, fuel consumption at $t$, fuel transported out from $m$ at $t$, and the arriving fuel at $t$.

\subsection{Co-optimization model for post-storm recovery}

For simplicity, we use a typical DC flow to model the power flow in power system. The co-optimization model for recovery scheduling is formulated as follows.

$$
\begin{aligned}
& \int \max \left[\alpha \sum_{i} \sum_{t} D_{i, t}+(1-\alpha) \sum_{r} \sum_{t} y_{r, t}\right] \\
& \text { s.t. } \quad(2)-(16) \\
& P_{i, t}-D_{i, t}=\sum_{l \in \mathcal{L}(i)} P L_{l, t} \quad \forall i, t \\
& P_{i, t} \leq P_{i, t}^{\max } \quad \forall i, t \\
& D_{i, t} \leq \hat{D}_{i, t} \quad \forall i, t \\
& -u_{l, t} \cdot P L_{l}^{\max } \leq P L_{l, t} \leq u_{l, t} \cdot P L_{l}^{\max } \quad \forall l, t \\
& -\left(1-u_{l, t}\right) M \leq P L_{l, t}-\frac{\theta_{i, t}-\theta_{j, t}}{X_{l}} \quad \forall l, t \\
& P L_{l, t}-\frac{\theta_{i, t}-\theta_{j, t}}{X_{l}} \leq\left(1-u_{l, t}\right) M \quad \forall l, t \\
& \theta_{1, t}=0 \quad \forall t
\end{aligned}
$$

where $D_{i, t}$ is the recovered load at node $i$ at time $t ; \hat{D}_{i, t}$ is the maximal load supplied at node $i ; P L_{l, t}$ is the power flow on line $l$ at time $t ; P L_{l}^{\max }$ is the maximum power flow on line $l ; X_{l}$ is the reactance of line $l$ connecting $i$ and $j$; and $\theta_{i}$ is the voltage angle at node $i$ at time $t$. 
The model optimizes the recovery scheduling considering the repair resource limitation and activity precedency relationship in the networked system. By solving the MILP problem above, we could determine the schedule of poststorm recovery for power and transportation system so that the load and road can be restored as much as possible over the scheduling periods.

\section{Case studies}

In the case study, the electricity distribution system is based on a simplified IEEE 13-node test feeder which is based a DC model, and the transportation system is illustrated in Fig. 2. DC power flow is solved for the IEEE 13-node feeder with two distributed generators (G1 and G2) at nodes 1, 7, and six loads (D1-D6) at nodes 4, 5, 8, 9, 11 , and 12, respectively. Electricity network nodes and transportation intersections co-located as shown in Fig. 2. The storm caused the closure of road 1-2 (R1-2), road 5-6 (R5-6), road 2-7 (R2-7), road 9-10 (R9-10) and road 12-13 (R12-13), generator outage at G1 and G2 (lack of fuel supply), and line outage at line 1-2 (L1-2) and line 7-8 (L78 ). The restoring resources/crew is dispatched from intersection 4 . The post-storm planning period is one day (24 hours) with one-hour time interval.

Two cases are studied: Case 1 only concerns about the total energy recovered in the short recovery period, which includes just the recovered energy terms in the objective function (i.e., $\alpha=1$ ); Case 2 concerns about both the recovered energy and the restored road. The results and related analysis are introduced in the following passage.

1) Case 1

By solving the co-optimization problem using the proposed method, the one-day optimized repair schedules for

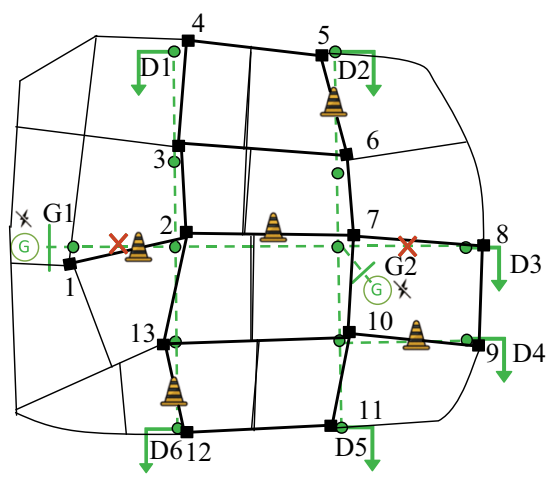

- - - Electricity distribution network; ○ Node; (G) Distributed generator — District boundary; — Roadway network; $\square$ Load; 具 Road closure $\nvdash$ Generator outage; $X$ Line outage; - Traffic intersection

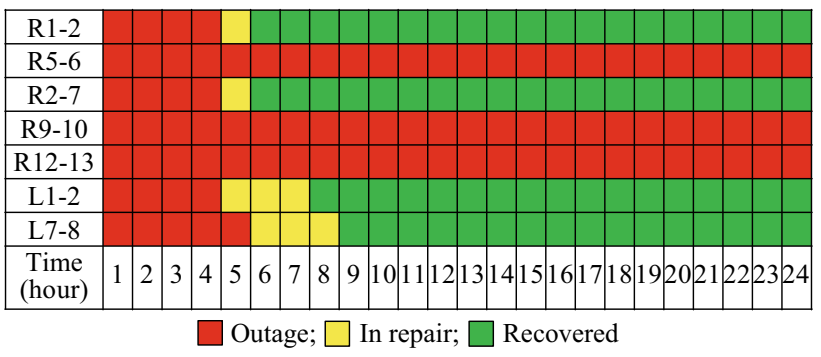

Fig. 3 Repair schedules for power and transportation systems in Case 1

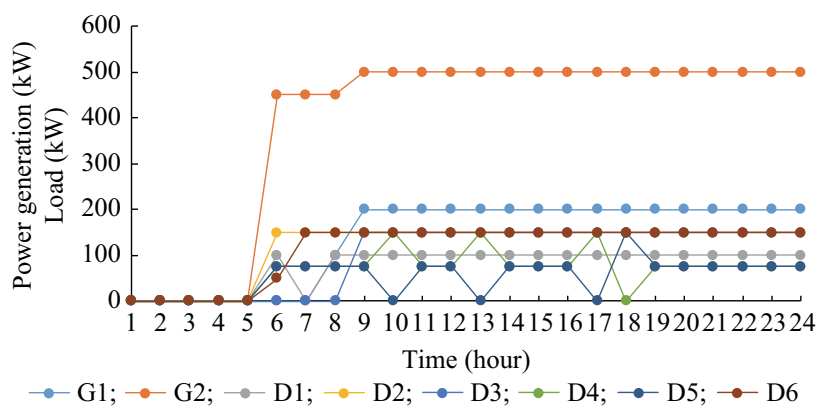

Fig. 4 Restored power generation and load in Case 1

power and transportation systems are obtained as Fig. 3, and the recovered power generation and load are demonstrated in Fig. 4.

The repair schedules demonstrate the starting time and completing (fully recovered) time to repair both the damaged roadways and the electricity lines. It is observed that R1-2 and R2-7 start to be repaired from hour 5, and at hour 6 , they are recovered to the normal status. The other three damaged roadways are not even repaired. With only two roadways recovered, both of the lines in outage are fixed as well as the two generators are restored. G2 is recovered earlier than G1 due to the characteristics of the topology. To recover $\mathrm{G} 2$ at node 7 , the resources can be delivered from intersection 4 to intersection 7 in different ways. At a result, G1 begins to generate power with the transported fuel at hour 6, right after the roadway R2-7 was recovered.

At the same time, all the loads are supplied with power from G2 except D3, which depends on the repair of L7-8. Right after L7-8 is recovered to the normal state, power is able to supply D3 at hour 9. Repair of G1 depends on the restoring of R1-2 and L1-2. Therefore, after both R1-2 and L1-2 are recovered at hour 8 (L1-2 recovered two hours later than R1-2, due to more restoring resources and crew required), G1 begins to generate and supply power to the loads at hour 8 . Hence, only with restoring roadways R1-2 and R2-7, the distribution system can be recovered.

Fig. 2 Post-storm interdependent power and transportation systems 
From the restored power and load curves, we notice there are a few hours when D4 and D5 are not supplied with power. That is the result of the fuel limit. If there is enough fuel for the power generation (fuel limits are removed), all the loads can be supplied continuously once the lines are restored. Therefore, the distribution system can be recovered and the most energy is restored with only a small part of infrastructure recovery in the transportation network through our proposed co-optimization method.

From the point of view of power system, the fast system recovery with the minimal efforts on the recovery of transportation system is desired. However, from the point of view of transportation system, the roadway recovery is also of great importance. Case 2 studies the scenario when the recovery of the both systems are regarded in the objective function.

\section{2) Case 2}

After co-optimization, the one-day optimized repair schedules for power and transportation systems are demonstrated in Fig. 5, and the recovered power generation and load are depicted in Fig. 6.

Different from the results of Case 1, in Case 2, all the roadways are repaired in the first half day. The repair schedules of five roadways are close to each other, therefore, repair multiple roadways simultaneously will result in the slower recovery time due to the limited repair resources and crew. Compared to only one hour restoring time for the two roadways in Case 1, Case 2 consumes a few more hours to fix each of the five roadways. Eventually at hour 12 , all the roads are cleared. Both electricity lines in outage are fixed at the same time as that in Case 1. Even more interesting, G1 and G2 begin to generate power at the same time as Case 1 as well. The six loads start to be supplied at the same time too. The only difference between the two cases is the variations of generation and supply levels. The total energy restored from the co-optimized results, nevertheless, are exactly the same in the two cases. It can be explained that, once the distribution system is recovered with the recovered lines and generators, the energy restored is only related to limits of fuel resources.

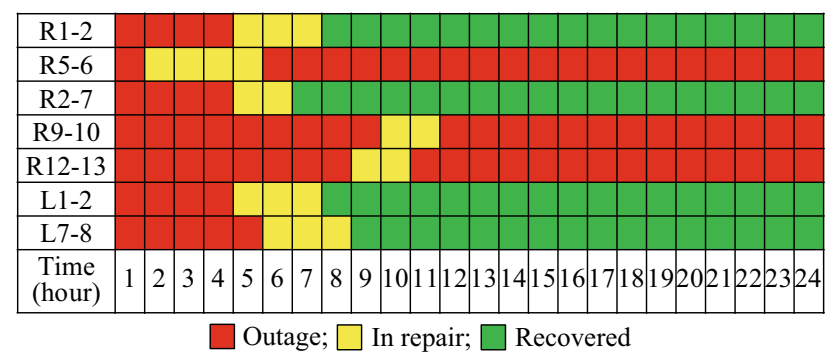

Fig. 5 Repair wchedules for power and transportation systems in Case 2

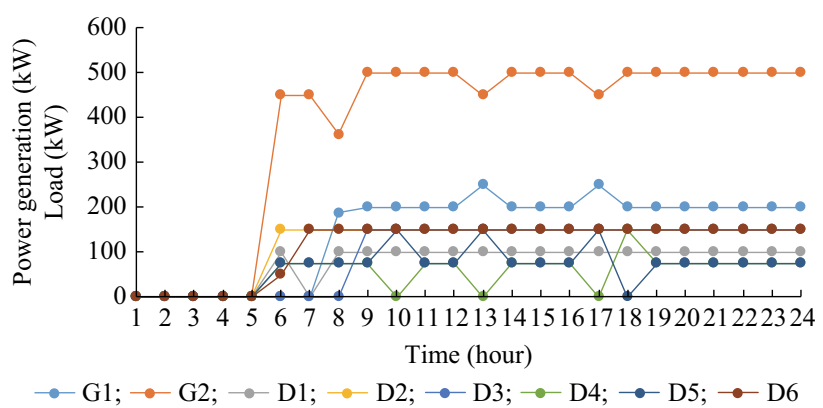

Fig. 6 Restored power generation and load in Case 2

In these two cases, the same recovery time is derived for distribution systems. For transportation system, however, Case 2 recovers faster than Case 1 due to the roadway recovery term in the objective function. Therefore, optimizing the recovery for both systems is more efficient, or at least preferred in this particular case study, as more infrastructures are expected to be restored in the short poststorm recovery time, which will reduce the loss to the most extent from the disaster.

\section{Conclusion}

This paper develops an integrative framework that addresses the challenges of enhancing the recovery efficiency for urban power and transportation systems in shortterm recovery period. We treat the post-storm recovery process as a scheduling problem with the constraints representing crew dispatch, equipment, fuel limit, and precedency relationship among the repair tasks. A new framework to co-optimize the repair scheduling for power and transportation systems is proposed. Two cases are studied, and the results are analyzed and compared. The amount of recovered electricity is concerned from the power system's perspective while the roadway recovery is also a crucial task from transportation's point of view. This study shows the importance of the interdependence of power and transportation systems in the recovery process and presents a novel co-optimization framework to address it. One of the future works is to consider more detailed constraints within this framework.

Acknowledgements This work was supported by the U.S. National Science Foundation Project (No. ECCS-171121), CARRER Award (No. CMMI-1554559), and CSU FRD-IoT award.

Open Access This article is distributed under the terms of the Creative Commons Attribution 4.0 International License (http:// creativecommons.org/licenses/by/4.0/), which permits unrestricted use, distribution, and reproduction in any medium, provided you give appropriate credit to the original author(s) and the source, provide a link to the Creative Commons license, and indicate if changes were made. 


\section{References}

[1] ABC9 (2017) Florida power outages from storm at 6.2 million. http://www.wftv.com/news/florida-power-outages-from-stormat-62-million-officials-say/605519895. Accessed 11 January 2019

[2] CNN (2017) The U.S. has been hit by two giant hurricanes: here's the financial toll. https://money.cnn.com/2017/09/10/ news/economy/hurricane-irma-harvey-economic-damage/index. html. Accessed 11 January 2019

[3] IPCC (2014) Climate change 2014: impacts, adaption, and vulnerability: part A global and sectoral aspects. https://www. ipcc.ch/report/ar5/wg2/. Accessed 11 July 2014

[4] Abbey C, Cornforth D, Hatziargyriou N et al (2014) Powering through the storm: microgrids operation for more efficient disaster recovery. IEEE Power Energy Mag 12(3):67-76

[5] Lopes JAP, Moreira CL, Madureira AG (2006) Defining control strategies for microgrids islanded operation. IEEE Trans Power Syst 21(2):916-924

[6] Morelato AL, Monticelli A (1989) Heuristic search approach to distribution system restoration. IEEE Trans Power Deliv 4(4):2235-2241

[7] Wang Z, Chen B, Wang J et al (2015) Coordinated energy management of networked microgrids in distribution systems. IEEE Trans Smart Grid 6(1):45-53

[8] Salman AM, Li Y, Stewart MG (2015) Evaluating system reliability and targeted hardening strategies of power distribution systems subjected to hurricanes. Reliab Eng Syst Saf 144:319-333

[9] Kwasinski A (2010) Technology planning for electric power supply in critical events considering a bulk grid, backup power plants, and micro-grids. IEEE Syst J 4(2):167-178

[10] lamonica M (2012) Microgrids keep power flowing through sandy outages. http://www.technologyreview.com/view/ 507106/microgrids-keep-power-flowing-through-sandyoutages/. Accessed 7 November 2012

[11] Strbac G, Hatziargyriou N, Lopes JP et al (2015) Microgrids: enhancing the resilience of the European megagrid. IEEE Power Energy Mag 13(3):35-43

[12] Executive Office of the President (2013) Economic benefits of increasing electric grid resilience to weather outages technical report. https://ieeexplore.ieee.org/document/6978705/. Accessed 9 August 2013

[13] Sun W, Liu CC, Zhang L (2011) Optimal generator start-up strategy for bulk power system restoration. IEEE Trans Power Syst 26(3):1357-1366

[14] Adibi MM, Fink LH (1994) Power system restoration planning. IEEE Trans Power Syst 9(1):22-28

[15] Florida Public Service Commission (2007) Report to the legislature on enhancing the reliability of Florida's distribution and transmission grids during extreme weather. https://www.docin. com/p-1615636411.html. Accessed 12 July 2007

[16] Wang Y, Chen C, Wang J et al (2016) Research on resilience of power systems under natural disasters: a review. IEEE Trans Power Syst 31(2):1604-1613

[17] Faturechi R, Miller-Hooks E (2014) Measuring the performance of transportation infrastructure systems in disasters: a comprehensive review. J Infrastruct Syst 21(1):1-15

[18] Chen Y, Tzeng G (2000) Determining the optimal reconstruction priority of a post-quake road-network. In: Proceedings of 8th international conference on computing in civil and building engineering (ICCCBE-VIII), Stanford, USA, 14-16 August 2000, pp 686-693

[19] Orabi W, El-Rayes K, Senouci A et al (2009) Planning postdisaster reconstruction efforts of damaged transportation networks. In: Proceedings of 2009 construction research congress, Seattle, USA, 5-7 April 2009, 7 pp

[20] Wakabayashi H, Iida Y (1992) Upper and lower bounds of terminal reliability of road networks: an efficient method with Boolean algebra. J Nat Disaster Sci 14(1):29-44

[21] Angeloudis P, Fisk D (2006) Large subway systems as complex networks. Physica A 367:553-558

[22] Chen L, Miller-Hooks E (2012) Resilience: an indicator of recovery capability in intermodal freight transport. Transp Sci 46(1):109-123

[23] Feng C, Wang T (2003) Highway emergency rehabilitation scheduling in post-earthquake 72 hours. J East Asia Soc Transp Stud 5(1):3276-3285

[24] Golroo A, Mohaymany A, Mesbah M (2010) Reliability based investment prioritization in transportation networks. In: Proceedings of 89th annual meeting of the transportation research board, transportation research board, Washington DC, USA, 12-14 September 2010, $14 \mathrm{pp}$

[25] He C, Wu L, Liu T et al (2017) Robust co-optimization scheduling of electricity and natural gas systems via ADMM. IEEE Trans Sustain Energy 8(2):658-670

[26] Li T, Eremia M, Shahidehpour M (2008) Interdependency of natural gas network and power system security. IEEE Trans Power Syst 23(4):1817-1824

[27] Liu C, Shahidehpour M, Fu Y et al (2009) Security-constrained unit commitment with natural gas transmission constraints. IEEE Trans Power Syst 24(3):1523-1536

[28] Zhang P, Qian K, Zhou C et al (2012) A methodology for optimization of power systems demand due to electric vehicle charging load. IEEE Trans Power Syst 27(3):1628-1636

[29] Lopes JAP, Soares FJ, Almeida PMR (2011) Integration of electric vehicles in the electric power system. Proc IEEE 99(1):168-183

[30] Deilami S, Masoum AS, Moses PS et al (2011) Real-time coordination of plug-in electric vehicle charging in smart grids to minimize power losses and improve voltage profile. IEEE Trans Smart Grid 2(3):456-467

Yinyin GE received the B.S. degree in automation and the M.S. degree in systems engineering from Xi' an Jiaotong University, China, in 2008 and 2011, respectively. She received the Ph.D. degree in electrical engineering at Illinois Institute of Technology, Chicago, USA, in 2016. She is currently a research associate at Case Western Reserve University, USA. Her research interests include power system resilience, PMU applications in smart grid, monitoring, visualization, and state estimation for distribution systems.

Lili DU received the B.S. degree in mechanical engineering from Xi'an Jiaotong University, China in 1998, the M.S. degree in industrial engineering from Tsinghua University, China in 2003, and the $\mathrm{Ph} . \mathrm{D}$. degree in decision sciences and engineering systems with a minor in operations research and statistics from Rensselaer Polytechnic Institute, USA in 2008. She worked as a post-doctoral research associate for NEXTRANS, the USDOT Region V Regional University Transportation Center at Purdue University, USA from 2008 to 2012. She is an associate professor in the Department of Civil and Coastal Engineering, University of Florida, USA. Before joining University of Florida, she worked as an assistant and then an associate professor at Illinois Institute of Technology, USA from 2012 to 2017. Her research is characterized by applying operations research, network modeling, and statistical methods into transportation system analysis and network modeling. 
Hongxing YE received the B.S. degree in electrical engineering, and the M.S. degree in systems engineering, both from Xi' an Jiaotong University, China, and the $\mathrm{Ph} . \mathrm{D}$. degree in electrical engineering from the Illinois Institute of Technology, Chicago, USA in 2016. He received Sigma Xi Research Excellence Award at Illinois Institute of
Technology in 2016. He is currently an assistant professor at Cleveland State University, USA. His research interests include power system optimization, electricity market, renewable integration, and cyber-physical system security in smart grid. 